\title{
Measuring chemical and electronic properties of individual nano-objects
}

\author{
D. Taverna, A. Vlandas, O. Stéphan, C. Colliex
}

\begin{abstract}
Laboratoire de Physique des Solides (UMR CNRS 8502), Bâtiment 510, Université Paris-Sud, 91405 Orsay (France)
\end{abstract}

The sub-nanometer probe of high energy (100-200 keV) electrons delivered by a field emission source in a Scanning Transmission Electron Microscope (STEM) column, constitutes a unique tool to investigate the local properties of a solid specimen. Among the different signals which can be simultaneously acquired, the Electron Energy Loss (EELS) spectra convey a wealth of useful information covering a wide spectral range from the $\mathrm{eV}$ to the $\mathrm{keV}$ domains. Between 5 and $25 \mathrm{eV}$ they typically concern the interband transitions above gap threshold as well as the collective response of conduction electrons. At higher energy losses, the characteristic electron excitations from well defined core orbitals exhibit fine structures quite sensitive to the local atomic environments.

Using the very efficient acquisition capabilities of new CCD-based parallel detectors, sequences of spectra connected to the successive positions of the incident probe, defined with very accurate spatial increments (typically a couple of angströms), constitute a new type of data set, defined as spectrum-images. Several processing routines have been developed for extracting the useful information from these huge sets of spatially resolved EELS spectra. The facility presently operating on the Orsay VG STEM offers optimum resolutions of the order of $0.3 \mathrm{eV}$ (after deconvolution) and $0.5 \mathrm{~nm}$ respectively along the spectral and the spatial axes. This instrumentation is therefore well suited to the exploration of the local composition and electronic properties of individual nano-objects or interfaces, visualized in parallel in the HAADF imaging mode.

A major use of this technique is to perform elemental analysis and mapping of nanostructures with a near-atomic spatial resolution. It has proved its efficiency in identifying coaxial morphologies within nanotubular objects [1] or evidencing azafullerene molecules of $\mathrm{C}_{48} \mathrm{~N}_{12}$ as the nuclei for the growth of $\mathrm{CN}_{\mathrm{x}}$ nano-onions linked together to form a material with highly resilient properties [2]. The sensitivity of this technique has been pushed to the limit of the identification of single metallic atoms trapped within $\mathrm{C}_{60}$ molecules piled along the core of single-walled carbon nanotubes [3]. These studies have used the standard background modelling and subtraction technique to measure the weight of the different chemical species within the analysed volumes. More elaborate methods have been implemented to cope for more complex situations. In particular MLS techniques, fitting each spectrum with a linear combination of reference ones, is quite efficient to map an element involved into different bonding states as demonstrated in figure 1. In this case, a complex specimen made of $\mathrm{B}, \mathrm{C}, \mathrm{N}$ and $\mathrm{O}$ based nanoparticles and nanotubes is analysed in terms of different chemical states of boron (metallic boron, $\mathrm{BN}$ and $\mathrm{B}_{2} \mathrm{O}_{3}$ ) using the fine structures of the $\mathrm{B} \mathrm{K}$ edge in these compounds. But EELS may offer quite other possibilities, such as discriminating surface and bulk plasmon modes in individual nanotubes [4] and nanowires with different diameters. Another example is shown in figure 2, where the shift of the atomic He 1s to $2 p$ atomic transition in bubbles of different diameters is monitored. This shift being related to the local atomic density, this method provides a route for mapping the gas pressure in nanometer-size bubbles [5]. The diversity of physical parameters (dielectric, optical, magnetic, transport, mechanical) which can be measured at the nanoscale using the richness of the information available in EELS spectra, is yet far from being fully recognized and identified, which will require further theoretical support. 
[1] K. Suenaga et al., Science 278 (1997) 653; Y. Zhang et al., Science 281 (1998) 973

[2] L. Hultman et al., Phys. Rev. Lett., 87 (2001) 225503

[3] K. Suenaga et al., Science 290 (2000) 2280

[4] O. Stéphan et al., Phys. Rev. B, 66 (2002) 155422

[5] D. Taverna et al., to be published (2003)

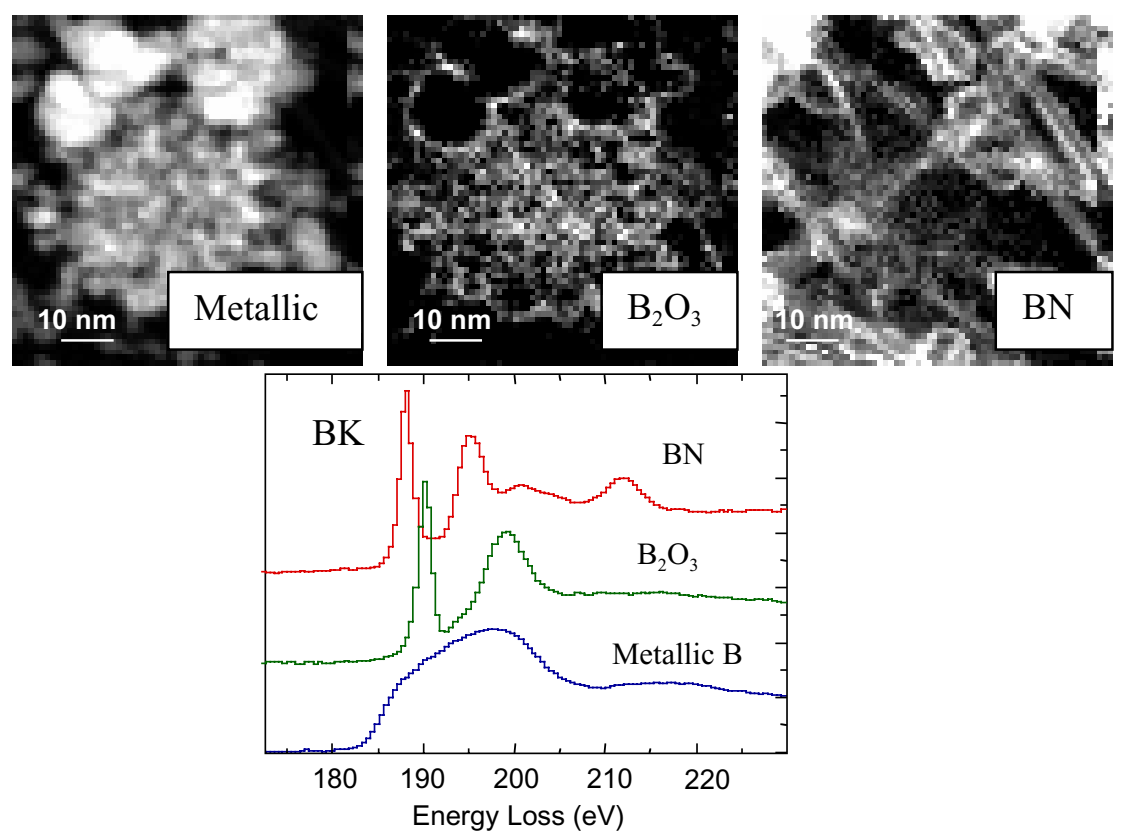

Figure 1 :Maps of boron in the different chemical states (metallic boron, $\mathrm{B}_{2} \mathrm{O}_{3}$ and $\mathrm{BN}$ ) in a nanoparticule and nanotube sample. $\mathrm{B} \mathrm{K}$ reference spectra used for reconstructing any experimental spectrum in the spectrum image.

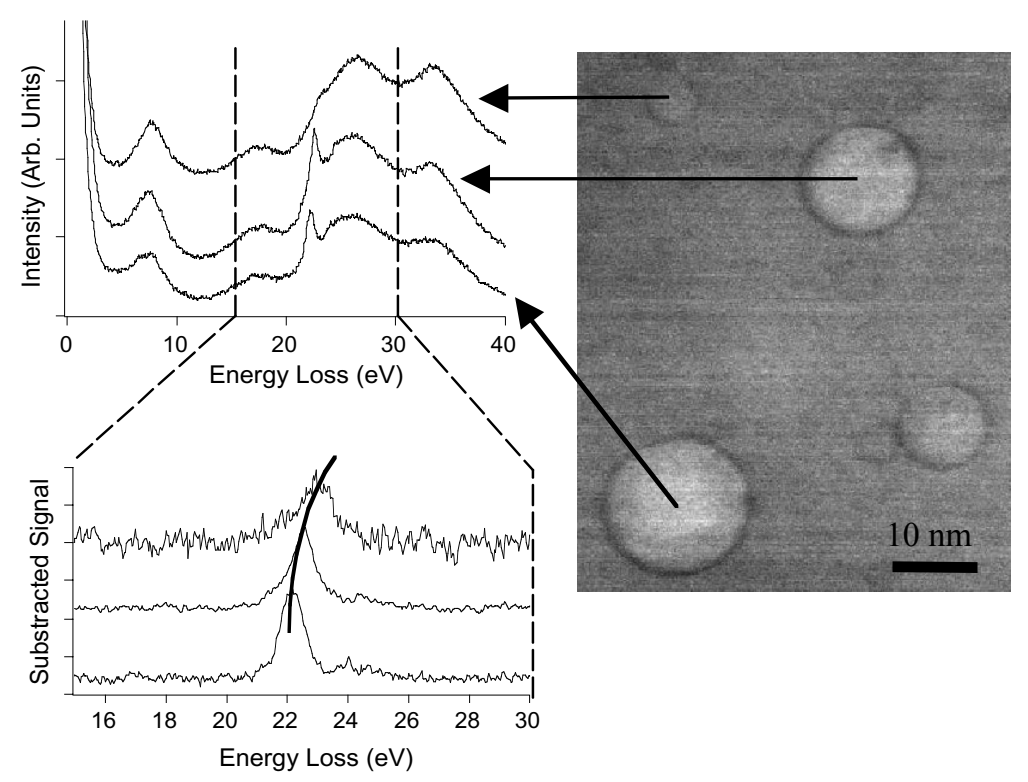

Figure 2 : Study of the shift of the He 1s line as a function of the size of He bubbles created in a $\mathrm{Pd}_{90} \mathrm{Pt}_{10}$ matrix aged in tritium atmosphere; all spectra have been acquired in the spectrum-image mode ( $64 \times 64$ pixels, $0.2 \mathrm{~ms}$ per pixel). The subtracted lines have required a refined modelling of the bulk energy loss function in the energy range around $22 \mathrm{eV}$ (sample CEA, coll. Univ. Bourgogne). 